Hier steht eine Anzeige.

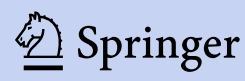


Einführung zum Thema

Gastroenterologe 2019 • 14:328-331

https://doi.org/10.1007/s11377-019-0378-6

(c) Springer Medizin Verlag GmbH, ein Teil von Springer Nature 2019

\author{
J. F. Riemann ${ }^{1}$ W. Fischbach ${ }^{2}$ \\ 'Stiftung LebensBlicke, Ludwigshafen, Deutschland \\ ${ }^{2}$ GastroLiga, Aschaffenburg, Deutschland
}

\title{
Sport und Krebserkrankungen
}

Es kann heute als gesichert gelten, dass körperliche Aktivität/Sport einen mittelbaren wie langfristig positiven Effekt auf die Entstehung und Ausprägung verschiedener Erkrankungen des Menschen hat. Gerade für Krebserkrankungen ist Sport ein essenzieller Faktor. Pro Jahr erkranken in Deutschland etwa 500.000 Menschen neu an Krebs, davon etwa 61.000 allein an Darmkrebs (KRK). Etwa 4 Mio. Menschen sind nach einer Krebserkrankung entweder geheilt oder leben als „cancer survivor“ mit einer medikamentösen Therapie lange Zeit bei guter Lebensqualität. Das vorliegende Heft versucht, aus verschiedenen Blickwinkeln den Einfluss von Sport bzw. körperlicher Aktivität auf Entstehung, Entwicklung und Verlauf von Krebserkrankungen zusammenzutragen.

Im 1. Beitrag werden sehr eindrücklich potenzielle molekulare und biologische Grundlagen dargestellt, die anschaulich zeigen, wo Einflussmöglichkeiten bestehen wie z. B. sportinduzierte Veränderungen auf Immunzellfunktionen und direkte Effekte auf das Tumormilieu. Wenngleich die Autoren einschränkend vermerken, dass es keine prospektiven Untersuchungen zu diesem Thema gibt, lassen doch viele nicht nur tierexperimentelle Studien erahnen, welche Wege möglich sind und wie in diesem Zusammenhang auf Krebserkrankungen eingewirkt werden kann.

Im 2. Beitrag werden wichtige epidemiologische Studien eingehend besprochen, die es derzeit zum Zusammenhang zwischen körperlicher Aktivität/Sport und gastrointestinalen Karzinomen gibt. Es zeigt sich übereinstimmend, dass Bewegung nicht nur präventiv wirkt, sondern vor allem auch postoperativ zu einer bedeutsamen Reduktion von Neuerkrankungen respektive von Rezidiven beitragen kann. Körperliche Aktivität/ Sport sind signifikant invers mit dem (Adeno-)Ösophagus- und Kardiakarzinom, KRK, Hepatozellulären Karzinom (HCC) und Pankreaskarzinom assoziiert.

Von daher ist eigentlich zwangsläufig, wie im 3. Beitrag belegt, dass Sport ein integraler Bestandteil der onkologischen Therapie sein sollte. Es darf heute als mangelnde Beratung durch behandelnde Ärzte gelten, wenn nach einer Krebserkrankung körperliche Bewegung/Sport nicht in ein Nachsorgeprogramm eingebaut oder zumindest nicht darauf hingewiesen wird, lassen sich doch krankheitsund therapiebedingte Belastungen deutlich reduzieren. Selbstverständlich müssen der körperliche Zustand sowie die Freiwilligkeit des Patienten berücksichtigt werden. Eine fachspezifische Betreuung in einem überwachten Setting, z. B. in Physiotherapieeinrichtungen, kann sehr hilfreich sein.

Krebs führt als chronische Erkrankung, wie sie derzeit aufgrund eines ständig sich weiter entwickelnden medikamentösen Therapiearsenals vor allem auch beim Kolonkarzinom angesehen werden kann, nicht selten zu außergewöhnlichen psychischen Belastungen. Ohne qualifizierte Hilfe, wie im 4. Beitrag gezeigt, wird manch ein Krebspatient einen dramatischen Einbruch seiner globalen Lebensqualität (Gefühlslage, Angst, Depressivität) erleben. Daher sind nicht nur psychoonkologische Hilfen notwendig. Auch der Sport bzw. körperliche Bewegung können zur Unterstützung beitragen. Verbesserte Versorgungskonzepte könnten ein Schlüssel zur bedarfsgerechten Versorgung von Patienten werden.

Im 5. Beitrag wird sehr eindrucksvoll am Beispiel des Netzwerks OnkoAk- 
tiv gezeigt, dass gezielte Hilfen zur Umsetzung entsprechender Bewegungsziele und eine Individualisierung der Sportund Bewegungstherapie in der Lage sind, Eindrucksvolles zu leisten. Es wäre $\mathrm{zu}$ wünschen, wenn es mehr solcher Aktivitäten gäbe. Das Konzept ist überzeugend; wir wissen aus eigener Erfahrung mit dieser Einrichtung, wie wichtig solche Hilfen sind. Körperliche Bewegung in einer Gemeinschaft bzw. unter Anleitung ist sicher förderlicher als so manche Einzelaktion.

Von abschließendem aktuellem Interesse ist der wichtige Beitrag zu Untersuchungen, die körperliche Bewegung/ Sport als sehr förderlich für die Phase vor Krebsoperationen einstufen. Faktoren wie Dauer des Krankenhausaufenthalts und Komplikationsraten können positiv beeinflusst werden. Prähabilitation könnte sich in Zukunft als ein weiteres Konzept erweisen, das den Umgang mit Krebserkrankungen erträglicher macht.

Das vorliegende Heft vermittelt nach unserer Überzeugung einen sehr kompetenten Einblick in die Notwendigkeit, körperliche Aktivität bzw. Sport einzusetzen im Sinne der Prävention, der Prä- und Rehabilitation von Krebserkrankungen sowie zur Verbesserung des Langzeitüberlebens bei guter Lebensqualität. Wir wünschen den Lesern viel Freude und hoffen, dass der ein oder andere Kollege in seiner täglichen Arbeit im Umgang mit Krebspatienten neue Anregungen für seine Patienten und für sich selbst finden wird.

J.F. Riemann, Ludwigshafen

W. Fischbach, Aschaffenburg

Im Juli 2019

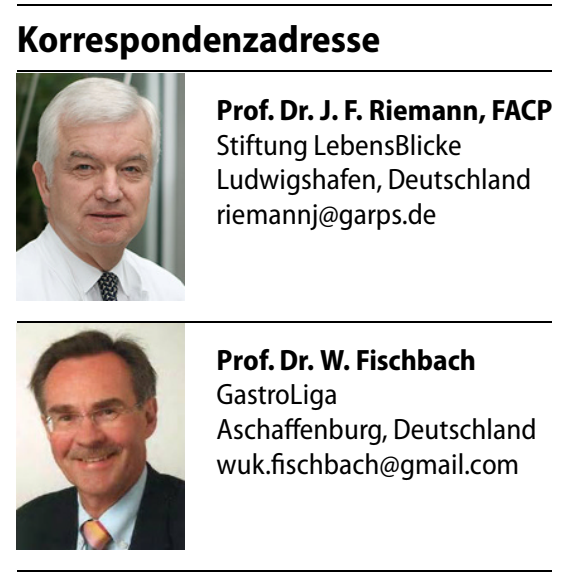

Interessenkonflikt. J.F. Riemann und W. Fischbach geben an, dass kein Interessenkonflikt besteht.
Förderpreis der Hans und Gertie Fischer-Stiftung

Ausschreibung

Im Rahmen der 194. Tagung der Rheinisch-Westfälischen Gesellschaft für Innere Medizin e.V. (RWGIM) in Dortmund am 29.11.30.11.2019 unter Leitung von Professor Labenz wird der Hans und Gertie Fischer Preis verliehen.

Bei diesem Preis handelt es sich um einen Förderpreis für die beste aus dem deutschsprachigen Raum vorgelegte Arbeit aus den Gebieten der Allgemeinen Inneren Medizin oder der Kardiologie. Für den Preis werden herausragende klinische oder experimentelle Originalarbeiten berücksichtigt, die sich der Erforschung der Ursachen, der Behandlung oder der Prävention von fachspezifischen Erkrankungen widmen.

Vorraussetzung für die Bewerbung: Bewerber sollen die Forschungsergebnisse an einer Klinik oder Forschungseinrichtung des Landes Nordrhein-Westfalen erarbeitet haben.

Der Preis ist mit 10.000,00 EURO dotiert, und kann ggf. auf mehrere Arbeiten aufgeteilt werden.

Eine Publikation der Arbeit in der vorgelegten Form oder in ihrem Wesentlichen Inhalt darf zum Zeitpunkt der Bewerbung um den Hans und Gertie Fischer-Preis nicht länger als 12 Monate zurückliegen. Die Bewerbungsfrist für den Hans und Gertie Fischer Preis 2019 endet mit dem 04.11.2019. Bewerber werden gebeten, ihre Arbeit in dreifacher Ausfertigung unter Angabe eines Kurztitels, sowie durch Beifügung eines kurzen Lebenslaufes und Autor/in-Referates bis zum 28. Oktober 2019 einzureichen an den Vorstand der Hans und Gertie Fischer-Stiftung

Herrn Prof. Dr. med. G. V. Sabin Chefarzt der Klinik für Kardiologie Städtische Kliniken Mönchengladbach $\mathrm{GmbH}$

Elisabeth-Krankenhaus Rheydt Kardiologie Sekretariat Hubertusstraße 100 41239 Mönchengladbach georg.sabin@sk-mg.de 
Hier steht eine Anzeige.

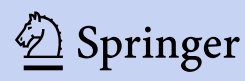

